H. D. Fegan

Nagoya Math. J.

Vol. 72 (1978), 83-92

\title{
THETA FUNCTIONS AND MODULAR JETS
}

\author{
H. D. FEGAN(1)
}

\section{Introduction}

Let $\Gamma$ be a subgroup of the modular group $\operatorname{PSL}(2, Z)$ then $\Gamma$ acts on the upper half plane $H=\{z \in C: \operatorname{Im} z>0\}$ and we can form the Riemann surface $M=H / \Gamma$, see [3]. The complex line bundles on a Riemann surface $M$ form a group $H^{1}\left(M, \mathcal{O}^{*}\right)$, see [4], and whenever we raise a line bundle to a power it will be in this group. Let $\kappa$ denote the canonical bundle on $M$ then a modular form of weight $\nu$ is a section of the bundle $\kappa^{k^{2 \nu}}$. A modular $n$-jet is then a section of $J^{n}\left(\kappa^{\frac{1}{2}}\right)$ the $n$-th jet bundle, see [7]. We can reformulate these ideas in the following terms. A modular form can be viewed as a function $\phi: H \rightarrow C$ and a modular $n$-jet as a vector valued function $\phi: H \rightarrow C^{n+1}$ both of which satisfy a transformation law under the elements of $\Gamma$.

By a theta function we shall mean a function of the form $\theta_{f}(z)$ $=\Sigma f(\lambda) \exp \left(i \pi\|\lambda\|^{2} z\right)$ where the summation is over those $\lambda$ lying in a lattice $L \subset \boldsymbol{R}^{\ell}, f$ is a polynomial in $\ell$ variables and $\theta_{f}: H \rightarrow C$ is a function on the upper half plane. We shall restrict ourselves to the case when $L=Z^{\ell}$ is the standard integer lattice and $f$ is homogeneous of degree 2n. By using the Fourier transform and Poisson summation formula we can express $\theta_{f}(-1 / z)$ as a sum of terms $a_{j}(z) \theta_{f_{j}}(z)$ for some polynomials $f_{j}$. This process can be iterated, applying it to $\theta_{f_{j}}$, and so we obtain a vector valued function $\left(\theta_{f}(z), \theta_{f_{j}}(z), \cdots\right)$ which has a transformation law under the map $z \rightarrow-1 / z$. Here the vector has possibly infinitely many entries. In fact this iteration produces no new theta functions after the first stage. More precisely we define a vector valued function $\theta_{f}(z)=\left(a_{r} \theta_{r}(z)\right)$ where $\theta_{f}: H \rightarrow C^{n+1}$ and the $r$-th co-ordinate is $a_{r} \theta_{r}(z)$. In this $\left\{a_{r}\right\}$ is a set of suitable constants and $\theta_{r}(z)=\theta_{f_{r}}(z)$ with essentially $f_{n-r}=\Delta^{r} f$. If $\Gamma(\theta)$ is the subgroup of $\operatorname{PSL}(2, Z)$ generated

Received July 11, 1977.

(1) The author was supported by the United Kingdom Science Research Council. 
by $z \rightarrow z+2$ and $z \rightarrow-1 / z$ then the question arises how $\theta_{f}$ behaves under the group $\Gamma(\theta)$. There is the following result.

THEOREM 1.1. The vector valued function $\theta_{f}(z)$ defines a modular jet.

We have already mentioned that modular jets can be viewed as vector valued functions with a certain transformation law and that theta functions give rise to vector valued functions with transformation laws. This theorem essentially says that these two transformation laws are the same. As a corollary of this we obtain immediately the well known result.

COROLLARY 1.2. The theta function $\theta_{f}(z)$ is a modular form if and only if $f$ is harmonic. If $f$ has degree $2 n$ then this form has weight $\frac{1}{2} \ell+2 n$ and multiplier $(-1)^{n}$.

This was proved in a special case by Hermite in 1858, see [2].

In their paper, see [6], Kuga and Shimura introduce the notion of a vector valued modular form. There are some similarities between their results and ours. In particular they obtain a decomposition for the space $\mathscr{F}_{n}$ of cusp $M_{n}$-forms. We describe our decomposition by giving a commutative diagram. The following notation is used; let $\Theta_{n}=\left\{\theta_{f}: \operatorname{deg} f=2 n\right\}, S_{n}=\left\{\theta_{f} \in \Theta_{n}: f\right.$ is harmonic $\}$ and let $J_{n}$ be the set of modular $n$-jets where all the functions have the form $\Sigma f(\lambda) \exp \left(i \pi\|\lambda\|^{2} z\right)$ on $H$.

THEOREM 1.3. The following diagram commutes

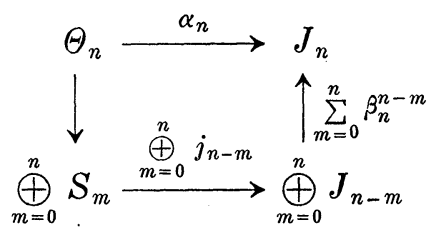

where the maps are as follows. The vertical map $\Theta_{n} \rightarrow \oplus_{m=0}^{n} S_{m}$ is defined by taking the decomposition into harmonic components of a polynomial. The map $\alpha_{n}$ is given by $\alpha_{n}\left(\theta_{f}\right)=\theta_{f}$, the modular $n$-jet defined by $\theta_{f}$ as in Theorem 1.1, and the maps $j_{n-m}: S_{m} \rightarrow J_{n-m}$ are given by taking $(n-m)$ jets. To give the maps $\beta_{n}^{m}$ we use local co-ordinates and then 


\section{$\beta_{n}^{m}\left(\phi_{0}, \cdots, \phi_{m}\right)={ }^{t}\left(0, \cdots, 0, \beta_{n, n-m}^{m} \phi_{0}, \cdots, \beta_{n, n}^{m} \phi_{m}\right)$ for suitable constants $\beta_{n, r}^{m}$. Precise details of these are given in section $V$.}

This result arises because there are two ways of obtaining a modular jet from a theta function. One way is to use the result of Theorem 1.1 directly and obtain a single modular $n$-jet. The other way is to decompose the polynomial of the theta function into harmonic pieces and then take the jet of each theta function which by Corollary 1.2 is a modular form. These must then be compared and the comparison is carried out by the maps $\beta_{n}^{m}$.

There are a number of differences between our result and that of Kuga and Shimura. Firstly the types of modular forms are different. In particular modular forms which are theta functions can under certain conditions, see [8], be written as the sum of an Eisenstein series and a cusp form and so can be modular forms which are not cusp forms. In addition the decomposition of [6] takes place in the space $\mathscr{F}_{n}$ of cusp $M_{n}$ forms while our decomposition is carried out at an earlier stage, that is in $\Theta_{n}$.

The methods which are used here can be adapted and used to study the heat equation on a Lie group. When we do this we obtain the asymptotic expansion of the trace of the heat kernel and direct proof of Kostant's form of Macdonald's $\eta$-functions, see [1] and [5].

\section{Modular jets}

We have indicated that a modular jet is a section of the $n$-jet bundle $J^{n}\left(\kappa^{\nu}\right)$ on $H / \Gamma$. However there are difficulties in taking the square root of a line bundle which appear in our case since we shall require the bundle $\kappa^{\frac{1}{4}}$. Now when $\Gamma=\Gamma(\theta)$ the Riemann surface $H / \Gamma$ is homotopy equivalent to a bouquet of circles, in fact the closed surface $\overline{H / \Gamma}$ is the sphere $S^{2}$. If $\xi$ is a line bundle on $M$ then there is a line bundle $\eta$ such that $\eta^{2}=\xi$ if and only if the Chern class $C(\xi)$ is even. On the closed Riemann surface $S^{2}$ it is a fact that $C(\kappa)=-2$, see [4], so the bundle $\kappa^{\frac{1}{2}}$ exists but $\kappa^{\frac{1}{4}}$ does not. However when we are considering line bundles on $H / \Gamma$ then since $H^{2}(H / \Gamma, Z)=0$ we have $C(\xi)=0$ for all line bundles and so we can find a line bundle $\kappa^{\frac{1}{2}}$. The difficulty here is that these bundles are not unique. In general if $M$ is a Riemann surface of genus $g$ then if $C(\xi)$ is even there are $2^{2 g}$ line bundles $\eta$ such that 
$\eta^{2}=\xi$. Thus on the closed surface $\overline{H / \Gamma^{\prime}}=S^{2}$ the square root of a line bundle is unique but we cannot find all the roots which we require while on the open surface $H / \Gamma$ all square roots exist but these are not unique.

To deal with these difficulties we shall work with functions on $H$. Let $\phi: H \rightarrow C$ be a modular form of weight $\nu$, so that $\phi(z+2)=\phi(z)$ and $\phi(-1 / z)=(z / i)^{\nu} \phi(z)$, with respect to $\Gamma(\theta)$. Notice that this is equivalent to giving a section of a line bundle on $H / \Gamma(\theta)$ and when we have to choose between different bundles we do this by specifying a section of the bundle. Define the vector valued function $\phi: H \rightarrow C^{k+1}$ by

$$
\phi(z)={ }^{t}\left(\phi(z), \phi^{\prime}(z), \cdots, \phi^{(k)}(z)\right)
$$

with $\phi^{(r)}(z)=(d / d z)^{r} \phi(z)$. Equation (2.1) defines an injection $\omega: J^{k}\left(\kappa^{\frac{1}{2} \nu}\right)$ $\rightarrow \operatorname{Maps}\left(H, C^{k+1}\right)$ by $\omega\left(j^{k} \phi(z)\right)=\phi(z), j^{k} \phi(z)$ denoting the equivalence class of functions having the same $k$-jet at $z$ as $\phi$. By the definition of jet bundles $\omega$ is independent of the choice of representative $\phi$ for $j^{k} \phi$. Define a matrix $A(z)=\left(a_{r s}(z)\right)$ for $0 \leq r, s \leq k$, by

$$
a_{r s}(z)= \begin{cases}\left(\begin{array}{l}
r \\
s
\end{array}\right)(\nu+s) \cdots(\nu+r-1) i^{r+s}(z / i)^{\nu+r+s} & \text { for } r>s \\
(-1)^{r}(z / i)^{\nu+2 r} & \text { for } r=s \\
0 & \text { for } r<s\end{cases}
$$

where $\left(\begin{array}{l}r \\ s\end{array}\right)$ is the binomial coefficient. Let $S: z \rightarrow-1 / z$ and $T: z \rightarrow z+2$ be generators of $\Gamma(\theta)$ and set $M(S, z)=A(z)$ and $M(T, z)=1$, the identity matrix. Since $S$ and $T$ generate $\Gamma(\theta)$ we have defined for each $z \in H$ an action of $\Gamma(\theta)$ on $C^{k+1}$.

Proposition 2.1. The vector valued function $\phi$ satisfies the transformation law $\phi(\gamma z)=M(\gamma, z) \phi(z)$ for $\gamma \in \Gamma(\theta)$.

Proof. We must show $\phi(\gamma z)=M(\gamma, z) \phi(z)$ for $\gamma=S$ and $\gamma=T$. This is clear for $\gamma=T$. For $\gamma=S$ we must show

$$
\phi^{(r)}(-1 / z)=\sum_{s=0}^{r}\left(\begin{array}{l}
r \\
s
\end{array}\right)(\nu+s) \cdots(\nu+r-1) i^{r+s}(z / i)^{\nu+r+s} \phi^{(s)}(z),
$$

which follows by induction and an elementary calculation, where $r$-th term in the right hand side should be read as $(-1)^{r}\left(\frac{z}{i}\right)^{\nu+2 r} \phi^{(r)}(z)$. 
Definition. Any vector valued function $f: H \rightarrow C^{k+1}$ satisfying the transformation law $f(\gamma z)=M(\gamma, z) f(z)$ will be called a modular $k$-jet.

\section{Theta functions}

The classical theta function $\theta(z)=\Sigma \exp \left(i \pi n^{2} z\right)$ satisfies the transformation laws

$$
\theta(z+2)=\theta(z) \text { and } \theta(-1 / z)=(z / i)^{\frac{1}{\theta}} \theta(z) .
$$

Here the square root is chosen so that if $z=i$ then $(z / i)^{\frac{1}{2}}=1$ and $(z / i)^{\frac{1}{2}}$ is single valued for $z \in H$. Equation (3.1) says that $\theta(z)$ is a modular form of weight $\frac{1}{2}$ for $\Gamma(\theta)$. We are interested in studying functions of the form $\theta_{f}(z)=\Sigma f(\lambda) \exp \left(i \pi\|\lambda\|^{2} z\right)$ when $f$ is a homogeneous polynomial of degree $2 n$. Notice that the restriction $\operatorname{deg} f=2 n$ is not a serious limitation since if $f$ has odd degree then

$$
\Sigma f(\lambda) \exp \left(i \pi\|\lambda\|^{2} z\right)=\Sigma f(-\lambda) \exp \left(i \pi\|-\lambda\|^{2} z\right)=-\Sigma f(\lambda) \exp \left(i \pi\|\lambda\|^{2} z\right) .
$$

Thus $\theta_{f}(z)=-\theta_{f}(z)$ and so $\theta_{f}(z)=0$ when the degree of $f$ is odd.

Changing notation replace $f$ by $f_{n}$ and define $f_{n-s}(\xi)=\Delta^{s} f_{n}(\xi) / 2^{s} s$ ! where $\Delta=\Sigma\left(\partial / \partial \xi_{j}\right)^{2}$ is the Laplacian and the indexing is chosen so that $\operatorname{deg} f_{r}=2 r$. Let $\theta_{r}(z)=\theta_{f_{r}}(z)$ and let the constant $a_{r}$ be

$$
a_{r}=(2 \pi i)^{r} r !(n-r) ! \nu(\nu+1) \cdots(\nu+r-1),
$$

with $\nu=\frac{1}{2} \ell$. The vector valued function $\theta_{f}$ is defined by

$$
\theta_{f}(z)={ }^{t}\left(a_{0} \theta_{0}(z), \cdots, a_{n} \theta_{n}(z)\right) .
$$

In the introduction in Theorem 1.1 we stated that $\theta_{f}$ gave rise to a modular jet. This is now stated more precisely as

THEOREM 3.1. The vector valued function $\theta_{f}$ has the transformation law $\theta_{f}(\gamma z)=M(\gamma, z) \theta_{f}(z)$ for $\gamma \in \Gamma(\theta)$ and $M(\gamma, z)$ the same as in Proposition 2.1.

Proof. Clearly $\theta_{f}(z+2)=\theta_{f}(z)$ and so the result is trivially true for $\gamma=T$. To complete the proof we must investigate how $\theta_{r}(z)$ transforms under $S: z \rightarrow-1 / z$. The method used to do this is to take Fourier transforms and use the Poisson summation formula.

Definition. If $g \in \mathscr{S}$, Schwartz space, the Fourier transform of $g$ 
is $\hat{g}(\xi)=\int_{-\infty}^{\infty} e^{2 \pi i \xi \cdot x} g(x) d x$. The Poisson summation formula is then

$$
\Sigma \hat{g}(\xi)=\Sigma g(n)
$$

To apply this we need the following result.

Proposition 3.2. Let $g(\lambda)=f(\lambda) \exp \left(i \pi\|\lambda\|^{2} z\right)$ then

$$
\hat{g}(\xi)=(z / i)^{-\frac{1}{2}(4 n+\ell)} i^{-2 n} \mathscr{H} f(\xi) \exp \left(-i \pi\|\xi\|^{2} / z\right),
$$

where $\mathscr{H}$ is the operator

$$
\mathscr{H} f(\xi)=\sum_{r=0}^{\infty} \Delta^{r} f(\xi) z^{r} /(-4 \pi i)^{r} r !
$$

Proof. In the special case $g(\lambda)=\exp \left(i \pi\|\lambda\|^{2} z\right)$ an elementary calculation shows that

$$
\hat{g}(\xi)=(z / i)^{-\frac{1}{2} \ell} \exp \left(-i \pi\|\xi\|^{2} / z\right) .
$$

Now let $f(\lambda)=\Sigma_{\alpha} a_{\alpha} \lambda^{\alpha}$ using multi-indices and $g(\lambda)=f(\lambda) \exp \left(i \pi\|\lambda\|^{2} z\right)$ then the Fourier transform is

$$
\hat{g}(\xi)=(2 \pi i)^{-2 n}(z / i)^{-\frac{1}{2} \ell} \Sigma_{\alpha} a_{\alpha}\left(\frac{1}{i} \frac{\partial}{\partial \xi}\right)^{\alpha} \exp \left(-i \pi\|\xi\|^{2} / z\right) .
$$

For convenience let $u=-2 \pi i / z$. We now introduce the Hermite polynomials with parameter $u$ and give some results about them.

Definition. The $k$-th Hermite polynomial with parameter $u$ is $h_{k}(x, u)=u^{-k} \exp \left(-\frac{1}{2} u x^{2}\right)(d / d x)^{k} \exp \left(\frac{1}{2} u x^{2}\right)$. With this definition we can write equation (3.3) in the form

$$
\hat{g}(\xi)=(2 \pi i)^{-2 n}(z / i)^{-\frac{1}{2} \ell} u^{2 n} \sum_{\alpha} a_{\alpha} h_{\alpha}(\xi, u) \exp \left(-i \pi\|\xi\|^{2} / z\right) .
$$

In this the notation is interpreted so that if $\alpha=\left(\alpha_{1}, \cdots, \alpha_{\ell}\right)$ then $h_{\alpha}(\xi, u)$ $=h_{\alpha_{1}}\left(\xi_{1}, u\right) \cdots h_{\alpha_{\ell}}\left(\xi_{\ell}, u\right)$. Thus we introduce the Hermite operator $\mathscr{H}$ on polynomials so if $f(\lambda)=\Sigma_{\alpha} a_{\alpha} \lambda^{\alpha}$ then $\mathscr{H} f(\xi)=\Sigma_{\alpha} a_{\alpha} h_{\alpha}(\xi, u)$. It is a fact, which will be shown later, that

$$
\mathscr{H} f(\xi)=\Sigma \Delta^{r} f(\xi) /(2 u)^{r} r ! .
$$

To complete the proof of Theorem 3.1 we apply the result of Proposition 3.2 and the Poisson summation formula to $\theta_{r}(z)$. Recall that

$$
\theta_{n-r}(z)=\Sigma_{\lambda} \Delta^{r} f(\lambda) \exp \left(i \pi\|\lambda\|^{2} z\right) / 2^{r} r !
$$


Hence

$$
\theta_{r}(z)=(z / i)^{-\frac{1}{2}(4 r+\ell)} i^{-2 r} \Sigma_{\xi} \mathscr{H}\left(\Delta^{n-r} f(\xi) / 2^{n-r}(n-r) !\right) \exp \left(-i \pi\|\xi\|^{2} / z\right),
$$

which upon substituting for $\mathscr{H}$ gives

$$
(2 \pi)^{r} \theta_{r}(-1 / z)=(z / i)^{\frac{1}{2} \ell} \sum_{s=0}^{r}\left(\begin{array}{l}
n-s \\
n-r
\end{array}\right)(-2 \pi)^{s}(z / i)^{r+s} \theta_{s}(z)
$$

Now let $a_{r}=(2 \pi i)^{r} r !(n-r) ! \nu(\nu+1) \cdots(\nu+r-1)$ with $\nu=\frac{1}{2} \ell$ then if $\theta_{f}(z)={ }^{t}\left(a_{0} \theta_{0}(z), \cdots, a_{n} \theta_{n}(z)\right)$ we find from equation (3.5) that

$$
\theta_{f}(-1 / z)=A(z) \theta_{f}(z)
$$

where $A(z)$ is defined in equation (2.2). This completes the proof of Theorem 3.1.

COROLlaRY 3.3. If $f$ is a homogeneous polynomial of degree $2 n$ then $\theta_{f}(z)$ is a modular form if and only if $f$ is harmonic and then this form has weight $2 n+\frac{1}{2} \ell$ and multiplier $(-1)^{n}$.

Proof. We apply equation (3.4) in the case $r=n$ and we find that $\theta_{f}(z)$ is a modular form if and only if $f$ is an eigenvalue of $\mathscr{H}$, in which case $\theta_{f}$ is a modular form with weight $2 n+\frac{1}{2} \ell$ and multiplier $(-1)^{n} c$, $c$ being the eigenvalue corresponding to $f$. It follows from the definition of $\mathscr{H}$ that $\mathscr{H} f=c f$ if and only if $c=1$ and $f$ is harmonic. By the multiplier of a modular form $\phi$ of weight $\nu$ we mean the constant $C$ such that

$$
\phi(-1 / z)=C(z / i)^{\nu} \phi(z)
$$

\section{The Hermite operator}

Recall the definition of the $k$-th Hermite polynomial with parameter $u, h_{k}(x, u)=u^{-k} \exp \left(-\frac{1}{2} u x^{2}\right)(d / d x)^{k} \exp \left(\frac{1}{2} u x^{2}\right)$. The results about there are easily proved and so we shall leave many of the details to the reader. The main result is the following.

LEMMA 4.1. $h_{k}(x, u)=\mathscr{H}\left(x^{k}\right)$ where $\mathscr{H} f=\Sigma_{r} \Delta^{r} f /(2 u)^{r} r !$ with $\Delta$ $=(d / d x)^{2}$.

Proof. It is easy to show that $h_{k}(x, \imath)$ satisfies the recurrence relation 


$$
u h_{k+1}(x, u)=\frac{d}{d x} h_{k}(x, u)+x u h_{k}(x, u)
$$

Now $h_{0}(x, u)=1$ and $h_{1}(x, u)=x$ so the result is trivially true for $k=0$ and 1. The proof can be completed by showing that $\mathscr{H}\left(x^{k}\right)$ satisfies the recurrence relation (4.1) and using induction.

Since the series defining $\mathscr{H}$ are exponential series we can apply this lemma one variable at a time to obtain the result

LEMMA 4.2. Let $\mathscr{H}$ denote the Hermite operator of the previous section, that is $\mathscr{H}\left(\Sigma_{\alpha} a_{\alpha} \lambda^{\alpha}\right)=\Sigma_{\alpha} a_{\alpha} h_{\alpha}(\xi, u)$, then if $f$ is a homogeneous polynomial in $\ell$ variables

$$
\mathscr{H} f(\lambda)=\Sigma \Delta^{r} f(\lambda) /(2 u)^{r} r ! .
$$

This result was used in the previous section.

We also used the fact that the homogeneous polynomial eigenfunctions of $\mathscr{H}$ were the harmonic polynomials and these have eigenvalue $c=1$. This follows most clearly when we consider how $\mathscr{H}$ is related to the grading on the space $V$ of polynomials. Let $V_{j}$ be the subspace of $V$ given by $V_{j}=\{f \in V: \operatorname{deg} f=j\}$. Now $\mathscr{H}$ preserves the associated filtration but not the grading. More precisely we have the following list of properties of $\mathscr{H}$. The natural projections of $V$ are denoted by $P_{r}: V \rightarrow V_{r}$.

Proposition 4.3. The Hermite operator $\mathscr{H}: V \rightarrow V$ satisfies the following.

a) $\mathscr{H}\left(\oplus_{j=0}^{k} V_{j}\right) \subset \bigoplus_{j=0}^{k} V_{j}$ for each $k$.

b) If $f \in V_{2 j}$ and $g \in V_{2 j+1}$ then

$$
P_{r} \mathscr{H} f=\left\{\begin{array}{ll}
f & \text { if } r=2 j \\
0 & \text { if } r \text { is odd }
\end{array} \quad P_{r} \mathscr{H} g= \begin{cases}g & \text { if } r=2 j+1 \\
0 & \text { if } r \text { is even } .\end{cases}\right.
$$

c) $\mathscr{H} \mid \oplus_{j=0}^{k} V_{j}$ is non singular for each $k$.

d) If $f$ is symmetric so is $\mathscr{H} f$.

Proof. These all follow from elementary calculations which are left to the reader.

The result about the eigenfunctions of $\mathscr{H}$ is now stated in the following.

COROLlaRY 4.4. Let $f$ be a polynomial eigenfunction of $\mathscr{H}$ so $\mathscr{H} f$ 
$=C f$ then $C=1$ and if $\operatorname{deg} f=n$ so $f=f_{n}+f_{n-1}+\cdots+f_{0}$ where $f_{j}$ is homogeneous of degree $j$ then $f_{j}$ is harmonic.

Proof. This follows by applying Proposition 4.3 to each of the components of $f$ in turn, starting with the highest $f_{n}$.

\section{The decomposition theorem}

In this section we shall prove Theorem 1.3. To begin we define the spaces we are considering; $\Theta_{n}=\left\{\theta_{f}: f\right.$ is homogeneous of degree $\left.2 n\right\}$, $S_{n}=\left\{\theta_{f} \in \Theta_{n}: f\right.$ is harmonic $\}$ and $J_{n}=\left\{\theta_{f}: \theta_{f} \in \Theta_{n}\right\}$. The result we shall prove is then

THEOREM 5.1. The following diagram commutes

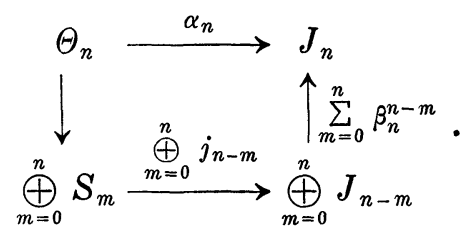

In this the map $\alpha_{n}$ is given by $\alpha_{n}\left(\theta_{f}\right)=\theta_{f}$. The vertical map $\Theta_{n}$ $\rightarrow \oplus_{m=0}^{n} S_{m}$ comes from the harmonic decomposition of $f$; more precisely if $f(\lambda)=\Sigma r^{2(n-m)} g_{m}(\lambda)$ where $r^{2}=i \pi\|\lambda\|^{2}$ and $g_{m}$ is harmonic and homogeneous of degree $2 m$ then $\theta_{f}(z) \rightarrow \Sigma \theta_{g_{m}}(z)$. The maps $j_{m}$ are defined by taking $m$-jets so $\Sigma \theta_{g_{m}}(z) \rightarrow \Sigma j_{n-m} \theta_{g_{m}}(z)$ where $j_{m} \phi={ }^{t}\left(\phi, \phi^{\prime}, \cdots, \phi^{(m)}\right)$. It only remains to define the map $\beta_{n}^{m}: J_{m} \rightarrow J_{n}$. Let

$$
\beta_{n, s}^{m}=\frac{(2 \pi i)^{n} m ! s ! \nu(\nu+1) \cdots(\nu+2 n-m-1)}{(s-n+m) !(\nu+s) \cdots(\nu+n+s-m-1)}
$$

where as before $\nu=\frac{1}{2} \ell$ then

$$
\beta_{n}^{m}\left(\phi_{0}, \cdots, \phi_{m}\right)={ }^{t}\left(0, \cdots, 0, \beta_{n, n-m}^{m} \phi_{0}, \cdots, \beta_{n, n}^{m} \phi_{m}\right) .
$$

The proof of Theorem 5.1 is essential a direct calculation. We shall indicate the main steps in this and leave the details to the reader. Firstly observe that it is sufficient to prove the result in the case $f(\lambda)$ $=r^{2(n-m)} g_{m}(\lambda)$ when the result becomes

$$
\theta_{f}(z)=\beta_{n}^{n-m} j_{n-m} \theta_{g_{m}}(z)
$$

This is sufficient since $\alpha_{n}\left(\theta_{f+g}\right)=\alpha_{n} \theta_{f}+\alpha_{n} \theta_{g}$ and $\theta_{f+g}=\theta_{f}+\theta_{g}$. Next 
observe that there is the formula

$$
\Delta r^{2 k} g_{m}=2 \pi i k(4 m+2 k+\ell-2) r^{2 k-2} g_{m},
$$

where $g_{m}$ is a harmonic polynomial homogeneous of degree $2 m$. This formula is a consequence of Euler's theorem from elementary analysis, namely $\lambda \cdot \operatorname{grad} g_{m}=2 m g(\lambda)$. The coefficients $\beta_{n, s}^{m}$ of the map $\beta_{n}^{m}$ were chosen to make the diagram commute and this can be checked directly by using the above two facts.

\section{REFERENCES}

[1] H. D. Fegan, The heat equation and modular forms, J. Diff. Geom., to appear.

[2] Ch. Hermite, Quelques formules realtives à la transformation des functions elliptiques, J. Math. Pures Appl., 3 (1858), 26-36.

[ 3 ] R. C. Gunning, Lectures on modular forms, Princeton University Press, Princeton, 1962.

[ 4 ] - Lectures on Riemann surfaces, Princeton University Press, Princeton, 1967.

[5] B. Kostant, On Macdonald's $\eta$-function formula, the Laplacian and generalized exponents, Advances in Math., 20 (1976), 179-212.

[6 ] K. Kuga and G. Shimura, On vector differential forms attached to automorphic forms, Jap. Math. Soc., 12 (1960), 258-270.

[ 7 ] R. S. Palais, Introduction to global non-linear analysis, Benjamin, New York, 1968. 1968.

[ 8 ] J. P. Serre, A course in arithmetic, Springer-Verlag, New York, 1973.

Rice University 